\title{
Two-step phase shifting in fringe projection: modeling and analysis
}

Jiaqi Mao, Yongkai Yin, Xiangfeng Meng, Xiulun Yang, Lei Lu, et al.

Jiaqi Mao, Yongkai Yin, Xiangfeng Meng, Xiulun Yang, Lei Lu, Dechun Li, Christian Pape, Eduard Reithmeier, "Two-step phase shifting in fringe projection: modeling and analysis," Proc. SPIE 10678, Optical Micro- and Nanometrology VII, 106780V (24 May 2018); doi: 10.1117/12.2306641

SPIE. Event: SPIE Photonics Europe, 2018, Strasbourg, France 


\title{
Two-step phase-shifting in fringe projection: Modeling and analysis
}

\author{
Jiaqi Mao ${ }^{a}$, Yongkai Yin*a,, Xiangfeng Meng, ${ }^{\mathrm{a}}$, Xiulun Yang ${ }^{\mathrm{a}}$, Lei $\mathrm{Lu}^{\mathrm{c}}$, Dechun $\mathrm{Li}^{\mathrm{a}}$, Christian Pape ${ }^{\mathrm{b}}$, \\ Eduard Reithmeier ${ }^{\mathrm{b}}$ \\ aShandong Provincial Key Laboratory of Laser Technology and Application, School of Information \\ Science and Engineering, Shandong University, Jinan 250100, China \\ ${ }^{\mathrm{b}}$ Institute of Measurement and Automatic Control, Leibniz University Hannover, Nienburger Str.17, \\ D-30167 Hannover \\ ${ }^{\mathrm{c} C o l l e g e}$ of Information Science and Engineering, Henan University of Technology, Zhengzhou \\ 450001, China
}

\begin{abstract}
This work proposes a two-step phase-shifting algorithm as an improvement of fringe projection profilometry. Considering the working process of fringe projection, the captured fringe image is formulated with two variables, i.e. surface reflectivity and phase value. And a phase shift of $3 \pi / 2$ is introduced to get the two-step phase-shifting. After appropriate variable substitution, expressions of two fringe images can be transformed into two equations corresponding to a line and a circle respectively. With this circle-line model, the characteristic of solution and the phase error due to non-zero ambient light are analyzed. Then the approach of error compensation is proposed based on estimation of the real fringe contrast and non-linear least square optimization. The validity of the proposed approach is demonstrated with both simulations and experiments.
\end{abstract}

Keywords: two-step phase-shifting, fringe projection profilometry, surface reflectivity, dynamic 3D shape measurement, phase-shifting profilometry

\section{INTRODUCTION}

With the advantages of being non-contact and non-destructive, optical three-dimensional (3D) shape measurement has been widely used in industrial manufacturing, computer vision, cultural heritage and many more ${ }^{1,2}$. There are numerous ways for optical 3D shape measurement, in which the fringe projection profilometry (FPP) has become one of the most popular methods in recent years ${ }^{3-5}$. With the development of FPP, improving the time efficiency of measurements has attracted a lot attention ${ }^{6-10}$. The framerate of a specific fringe projection system is a limiting factor and determined by the hardware. For certain hardware configuration, improvement of time efficiency mainly depends on reducing the number of fringe patterns needed for a single measurement. Theoretically, single-shot methods ${ }^{11}$ based on different transforms ${ }^{12}$, ${ }^{13}$ are the most time efficient methods. However, phase calculation with single fringe image suffers from spectrum leakage and is difficult with complex surfaces. Therefore, phase-shifting approaches are frequently applied in FPP to improve the robustness and accuracy of phase calculation ${ }^{14-16}$.

*yinyongkai@sdu.edu.cn

Optical Micro- and Nanometrology VII, edited by Christophe Gorecki, Anand Krishna Asundi, Wolfgang Osten, Proc. of SPIE Vol. 10678, 106780V · (c) 2018 SPIE CCC code: $0277-786 X / 18 / \$ 18 \cdot$ doi: $10.1117 / 12.2306641$ 
In general, a minimum of three phase-shifted patterns are required to retrieve the phase information ${ }^{17}$, since the reconstruction equation has three independent variables. To further reduce the number of fringe patterns for a single measurement, the two-step phase-shifting approach based on elimination of the background term is investigated. An intuitive method to eliminate the background term is subtraction between two $\pi$-out-of-phase sinusoidal fringe patterns. Then the phase can be calculated directly with inverse cosine ${ }^{18}$. Another approach is to use the Hilbert transform to obtain the in-quadrate component of the processed fringe pattern, with which the phase can be calculated ${ }^{19}$. If the background intensity of the fringe patterns is slowly changing, it can be removed via an intensity differential algorithm. In this case the phase can be calculated using two fringe patterns with phase shift of $\pi / 2^{20}$. The background term can also be regarded as a direct current (DC) term, thus the phase can be calculated with arbitrary phase shift in $(0, \pi)$ after DC-term suppression ${ }^{21}$.

However, all the existing methods are proposed based on the general expression of the fringe projection with three variables. A novel two-step phase-shifting algorithm is proposed in this paper. Considering the working process of fringe projection, the captured fringe image is formulated with only two variables, i.e. surface reflectivity and phase value. Therefore, the phase can be calculated in principle with two fringe images. When the phase shift of $3 \pi / 2$ is introduced, the solution of the equations can be well demonstrated with the intersection between a line and a circle. With this circle-line model, the characteristic of solution and the phase error due to non-zero ambient light are analyzed. Then the approach of error compensation is proposed based on contrast estimation and non-linear least square optimization. The paper is arranged as follows: Section 2 is the principle and some theoretical analysis of proposed approach; section 3 is the results and discussions of both simulation and experiment; section 4 makes a conclusion for this paper.

\section{PRINCIPLE AND ANALYSIS}

\subsection{Two-step phase-shifting algorithm}

In the classical fringe projection profilometry, the intensity of the deformed sinusoidal fringe after reflected from the object surface can be written as

$$
I(x, y)=A(x, y)+B(x, y) \cos [\phi(x, y)]
$$

where $I(x, y)$ is the intensity of the captured image at pixel position $(x, y), A(x, y)$ is the background intensity, $B(x, y)$ represents the amplitude intensity and $\phi(x, y)$ denotes the phase distribution. There are three unknown variables $A, B$ and $\phi$ in the above formulation, thus at least three equations are needed to determine the unknown values, which leads to the well-known three-step phase-shifting. Here we try to discuss the possibility of two-step phase-shifting based on the process of fringe projection profilometry.

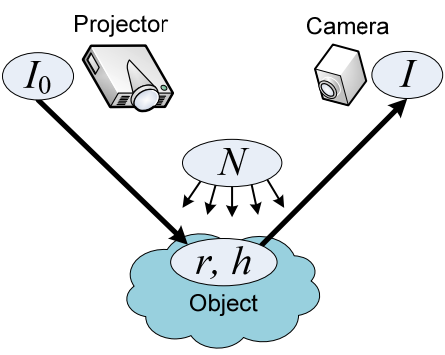

Figure 1. The process of the fringe projection profilometry. 
A standard sinusoidal fringe pattern generated by computer can be described as

$$
I_{0}(x, y)=a\left\{1+A_{m} \cos \left[\phi_{0}(x, y)\right]\right\}
$$

where $\phi_{0}(x, y)$ denotes the standard phase distribution. $a$ is the background term and $A_{m}$ is the contrast of fringe, both of them are set before fringe generation. They are all constants and will not change with the variation of the location. As shown in Fig. 1, after $I_{0}$ is projected onto the surface of the object via the projector, the camera captures the deformed fringe $I(x, y)$, which is reflected from the surface. During this process, the surface reflectivity $r(x, y)$ can proportionally change the intensity of fringe, while the surface shape $h(x, y)$ has an implicit impact on phase distribution. In addition, the ambient light $N(x, y)$ may also affect the final intensity of the fringe. Therefore, the deformed fringe recorded by the camera can be formulated as

$$
I(x, y)=\operatorname{ar}(x, y)\left\{1+A_{m} \cos [\phi(x, y)]+N(x, y)\right\}
$$

If the system is working in the darkness, the ambient light can be ignored. Then the deformed fringe can be simplified into

$$
I(x, y)=R(x, y)\left\{1+A_{m} \cos [\phi(x, y)]\right\}
$$

where $R(x, y)=\operatorname{ar}(x, y)$ and it is proportional to the reflectivity of the surface, so we still call it reflectivity. There remain only two unknown variables in the aforementioned expression and only two equations are needed to solve them in theory. Therefore, two fringes of two-step phase-shifting may be sufficient to calculate the phase.

Assume the shift of phase between two fringes is $\Delta \phi=3 \pi / 2$, then the corresponding equations for two-step phase-shifting algorithm can be formulated as follows

$$
\left\{\begin{array}{l}
I_{1}(x, y)=R(x, y)\left\{1+A_{m} \cos [\phi(x, y)]\right\} \\
I_{2}(x, y)=R(x, y)\left\{1+A_{m} \sin [\phi(x, y)]\right\}
\end{array}\right.
$$

After dividing $I_{1}(x, y)$ by $I_{2}(x, y)$, an equation only related to the phase is obtained

$$
\frac{I_{1}(x, y)}{I_{2}(x, y)}=\frac{1+A_{m} \cos [\phi(x, y)]}{1+A_{m} \sin [\phi(x, y)]}
$$

According to the character of trigonometry, the distribution of phase $\phi(x, y)$ can be solved. To simplify the expression of solving process, we take the following three variable substitutions

$$
k=\frac{I_{1}(x, y)}{I_{2}(x, y)}, \quad s=A_{m} \cos [\phi(x, y)], \quad t=A_{m} \sin [\phi(x, y)]
$$

Then Eq. (6) turns into the following form

$$
\left\{\begin{array}{l}
t=\frac{1}{k}(s+1)-1 \\
s^{2}+t^{2}=A_{m}{ }^{2}
\end{array}\right.
$$

The phase $\phi=\arctan (t / s)$ of each pixel can be obtained after solving $s$ and $t$ from these equations. With the knowledge of analytic geometry, Eq. (8-a) corresponds to a line $l$ while Eq. (8-b) a circle $O$. The solution of Eqs. (8) can be regarded as 
the intersection between the line and the circle, as shown in Fig. 2. And $\phi$ happens to be the vector angle of the intersection. It should be noticed that the line $l$ always goes through the point $(-1,-1)$ and its slope is $1 / k$. The circle $O$ is centered at the origin and its radius is $A_{m}$.

Generally, Eqs. (8) has two possible solutions, which correspond to two phase values $\phi_{1}$ and $\phi_{2}$. While exception appears when the line is tangent to the circle. In this case, $k$ reaches its extreme value and the tangent point $P_{a}$ or $P_{b}$ is the single solution of Eqs. (8), which corresponds to the phase value $\phi_{a}$ or $\phi_{b}$. Since the phase value is wrapped within the interval $(-\pi, \pi]$, it can be inferred that $\phi_{1}$ and $\phi_{2}$ will be in a specific range respectively, i.e., $\phi_{1} \in\left(-\pi, \phi_{b}\right] \cup\left(\phi_{a}, \pi\right]$ and $\phi_{2} \in\left(\phi_{b}, \phi_{a}\right]$. For the fringe within one period, the real phase values must be selected from $\phi_{1}$ or $\phi_{2}$, in which case the selected phase values can make a full distribution within $(-\pi, \pi]$. The correct phase value can be selected by exploiting the monotonicity of phase value during one period.

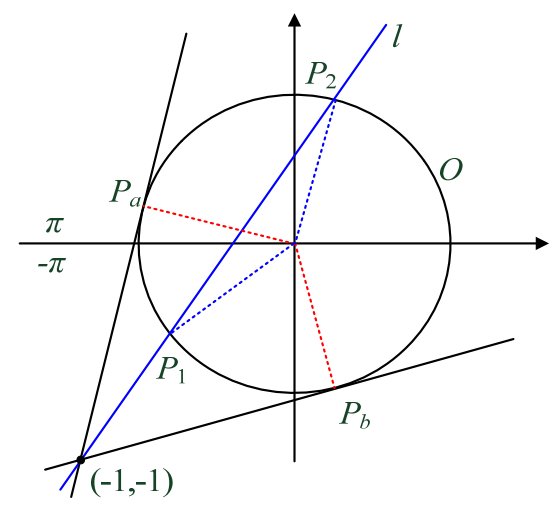

Figure 2. Illustration of the solution.

\subsection{Error analysis and compensation}

In practical applications, it is difficult to totally avoid the disturbance of ambient light. There always exists active ambient light arising from the structured illumination of the projector. When the fringe patterns are projected, the light may be diffused and reflected multiple times on the surface of the object, which leads to the ambient light, especially for the object with complex surface. Moreover, the projectors minimum intensity is larger than zero, even locations where the projected pattern is zero. In addition, the smooth filtering is commonly applied to the captured fringe images, which aims to suppress the random noise, but it will reduce the fringe contrast at the same time. And there will be passive ambient light if the experimental environment is not completely dark. Therefore, it is necessary to further discuss the influence of ambient light.

Assuming that the intensity of the ambient light is smooth, the intensity of ambient light can be treated as locally constant, which is indicated by $N$. The modified expression of deformed fringe is as follows

$$
I(x, y)=R(x, y)\left\{1+A_{m} \cos [\phi(x, y)]+N\right\}
$$

This expression can be transformed into a similar form with Eq. (4)

$$
I(x, y)=R^{\prime}(x, y)\left\{1+A_{m}^{\prime} \cos [\phi(x, y)]\right\}
$$


where $R^{\prime}(x, y)=R(x, y)(1+N)$ and $A_{m}^{\prime}=A_{m} /(1+N)$. It should be noticed that $A_{m}^{\prime}<A_{m}$, which means the fringe contrast decreases under the impact of ambient light. For Eq. (10), its phase can still be solved by the afore-mentioned two-step phase-shifting algorithm. The difference is that the radius of circle corresponding to Eq. (10) becomes smaller, as shown in Fig. 3(a). If $A_{m}$ is still used to calculate the phase with Eqs. (8), the calculated phase will have an error $\delta \phi$ comparing with the true value. It can be found that the error is periodic and will reach its maximum absolute value when $k$ reaches its extreme value, which corresponding to $\phi_{a}^{\prime}$ or $\phi_{b}^{\prime}$. And at that time the sign of the error will change because the selection of correct phase value is switched between $\phi_{1}$ and $\phi_{2}$. The distribution of phase error is schematically shown in Fig. 3(b).

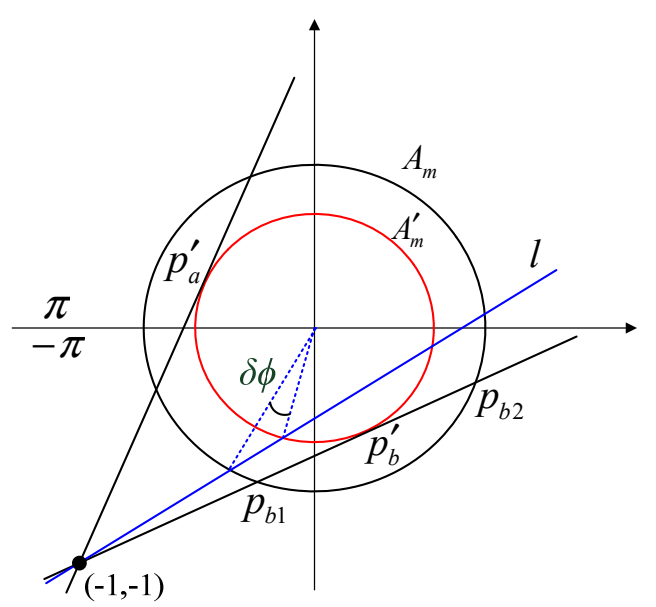

(a)

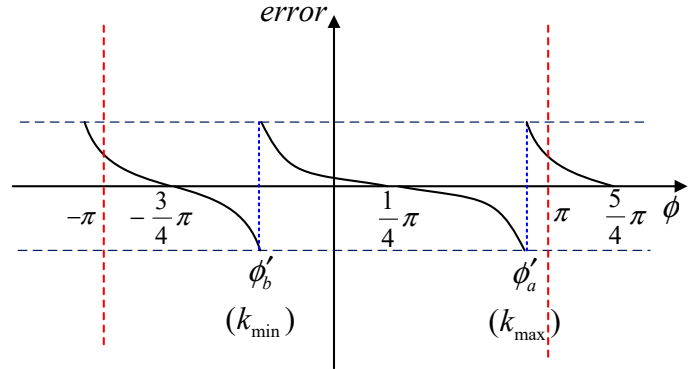

(b)

Figure 3. Influence of non-zero ambient light. (a) Decrease of fringe contrast leads to phase error, (b) The periodic distribution of phase error.

To compensate the phase error, the actual fringe contrast $A_{m}^{\prime}$ needs to be estimated. When $k$ takes the minimum value, Eqs. (8) will give two solutions corresponding to $P_{b 1}$ and $P_{b 2}$ shown in Fig. 3(a), with which $A_{m}^{\prime}$ can be calculated. $A_{m}^{\prime}$ corresponding to the maximum value of $k$ can also be calculated with a similar approach. According to the assumption of the smoothness of the ambient light, $A_{m}^{\prime}$ of any pixel in the fringe image can simply take the value of $A_{m}^{\prime}$ corresponding to the closest extreme. A more accurate phase is available when the new fringe contrast $A_{m}^{\prime}$ is substituted into Eqs. (8).

However, due to the discrete sampling of digital image, the selected extreme of $k$ still deviates from its analytical solution, which retains the error in the subsequent contrast estimation and phase calculation. To estimate the phase of the pixel which is close to the extreme of $k$ more accurately, we treat $A_{m}^{\prime}$ as a variable and try to solve $A_{m}^{\prime}$ and $\phi$ directly. In this case, the two-step phase-shifting for these pixels becomes an underdetermined problem, since there are three variables now. However, the local phase could be approximated to evolve linearly ${ }^{22}$, which will introduce a new constraint

$$
\phi_{a b s}=\phi_{c}+p \Delta \phi_{p}
$$

where $p$ is the distance to the central pixel, $\phi_{c}$ is the phase of the center pixel and $\Delta \phi_{p}$ indicates the phase difference between adjacent pixels. Then for all the pixels within a neighborhood, we have 


$$
\frac{I_{1}(p)}{I_{2}(p)}=\frac{1+A_{m}^{\prime} \cos \left(\phi_{c}+p \Delta \phi_{p}\right)}{1+A_{m}^{\prime} \sin \left(\phi_{c}+p \Delta \phi_{p}\right)}
$$

If the number of pixels taken is larger than 3 , it will become an over-determined problem, which can be well solved with the following non-linear least-squares optimization

$$
\min _{A_{m}^{\prime}, \phi_{c}, \Delta \phi_{p}} \sum_{p=-N}^{N}\left\{I_{1}(p) \times\left[1+A_{m}^{\prime} \sin \left(\phi_{c}+p \Delta \phi_{p}\right)\right]-I_{2}(p) \times\left[1+A_{m}^{\prime} \cos \left(\phi_{c}+p \Delta \phi_{p}\right)\right]\right\}^{2}
$$

Since the optimization will dramatically increase the time cost, this phase rectification is only applied to the pixel close to the extreme of $k$, where they have larger error comparing with other pixels.

\section{SIMULATIONS AND EXPERIMENTS}

\subsection{Simulation results}

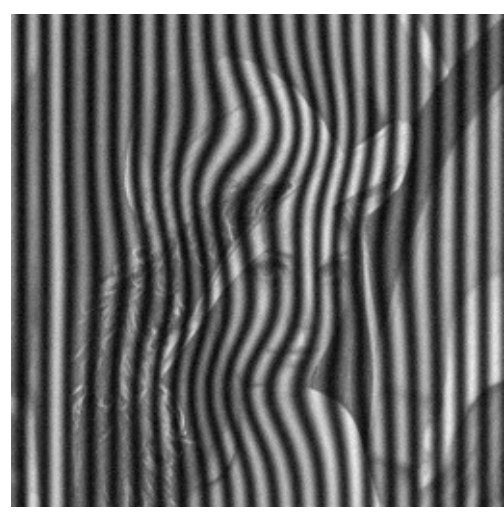

(a)

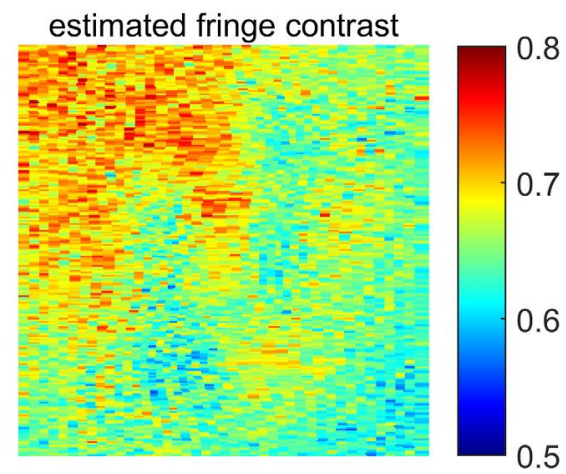

(c)

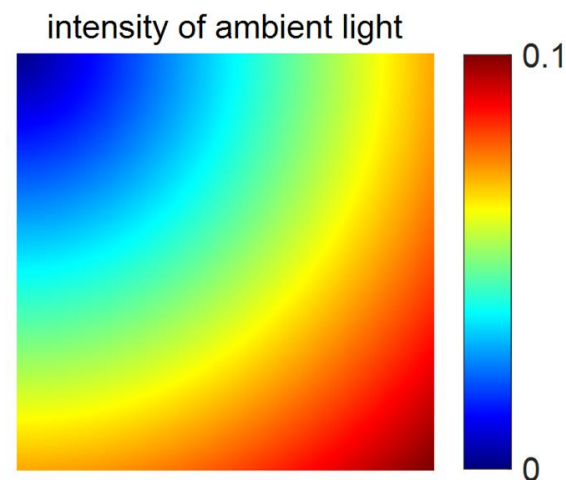

(b)

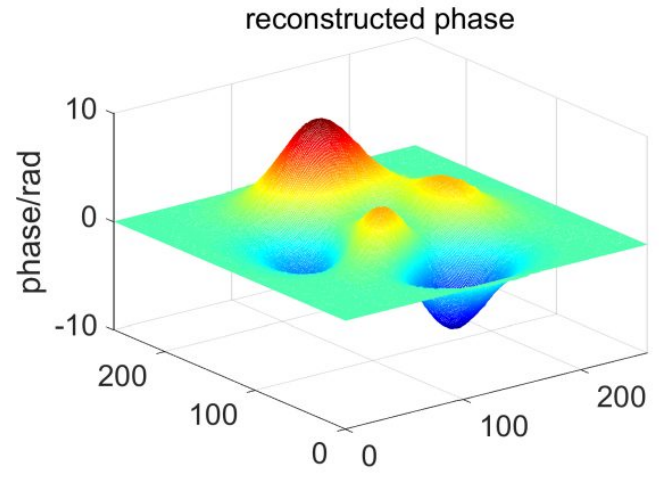

(d)

Figure 4. Simulation results. (a) One of simulated fringe images, (b) The intensity distribution of ambient light, (c) The estimated fringe contrast, (d) The reconstructed unwrapped phase after removing the carrier phase. 
Simulations are performed to evaluate the validity of the proposed algorithm. The deformed fringe images are generated with the following formulations

$$
\begin{aligned}
& I_{1}(x, y)=\operatorname{ar}(x, y)\left\{1+A_{m} \cos [2 \pi f x+\phi(x, y)]+N(x, y)\right\} \\
& I_{2}(x, y)=\operatorname{ar}(x, y)\left\{1+A_{m} \sin [2 \pi f x+\phi(x, y)]+N(x, y)\right\}
\end{aligned}
$$

The parameter settings are set to $a=0.5, A_{m}=0.8$ and $f=1 / 12$. The non-uniform reflectivity $r(x, y)$ is simulated with the Lena image whose intensity range has been compressed to $[0.4,1]$. The phase distribution $\phi(x, y)$ is simulated with a surface function of two variables, and the ambient light $N(x, y)$ is simulated with another surface function. The white Gaussian noise is added into the images to simulate the noise of camera. Figure 4(a) shows one of the generated fringe images, and Fig. 4(b) shows the intensity distribution of added ambient light, in which the left-top corner has no ambient light while the right-bottom corner has the largest ambient light intensity. Figure 4(c) shows the estimated fringe contrast $A_{m}^{\prime}$, which corresponds to the distribution of the ambient light. The unwrapped phase after the final optimization is shown in Fig. 4(d), from which the carrier phase denoted by $2 \pi f x$ has been removed.

(a)

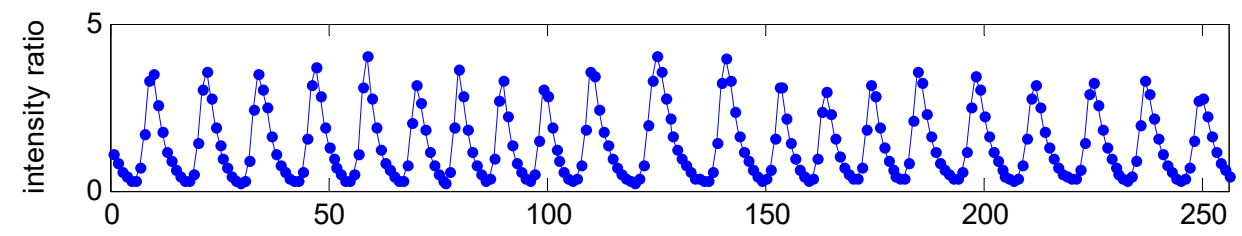

(b)

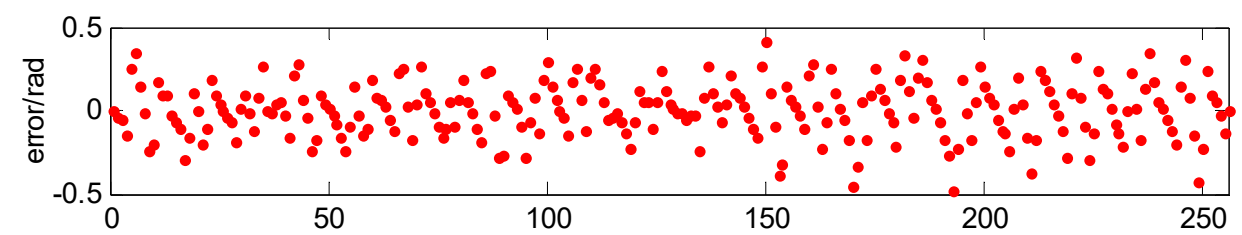

(c)

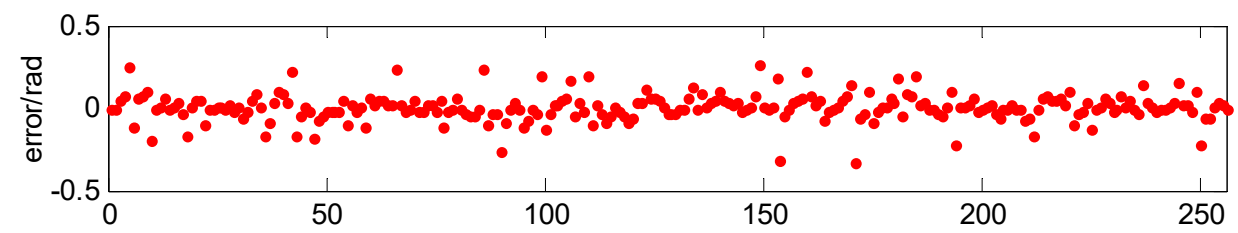

(d)

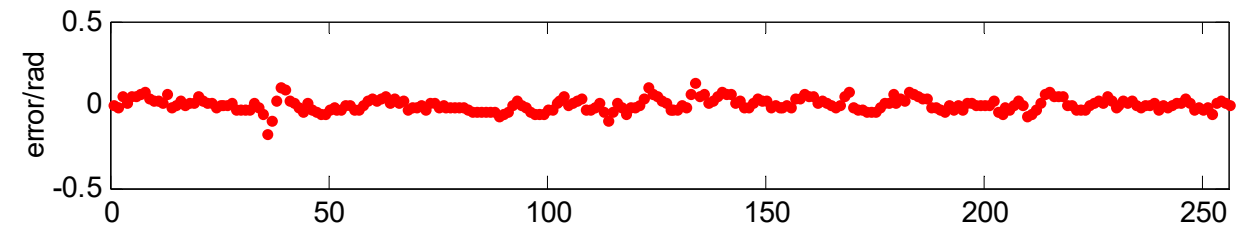

Figure 5. The intensity ratio and phase error distributions corresponding to the $100^{\text {th }}$ row of the image. (a) Intensity ratio between $I_{1}$ and $I_{2}$, (b) Phase error corresponding to given fringe contrast $A_{m}$, (c) Phase error corresponding to estimated fringe contrast $A_{m}^{\prime}$, (d) Phase error corresponding to final rectification using non-linear least square optimization.

As the real phase distribution is known in the simulation, we can well analysis the phase error of proposed algorithm. The phase error distributions corresponding to different stages of proposed approach are comparatively shown in Fig. 5. To make the demonstration clearer, only the data corresponding to the $100^{\text {th }}$ row of the image is shown. And the intensity ratio $k$ between $I_{1}$ and $I_{2}$ is displayed in Fig. 5(a) to provide positioning reference on $x$-axis. The phase error corresponding to given fringe contrast $A_{m}$ is shown in Fig. 5(b), which can be seen the non-zero ambient light introduces 
significant errors. The larger errors always appear in the same positions as the extreme of $k$, which is consistent with the analysis in section 2.2. The phase errors corresponding to estimated fringe contrast $A_{m}^{\prime}$ and final rectification using non-linear least square optimization are shown in Fig. 4(c) and 4(d) respectively, which show dramatical reduction in the error respectively. The root-mean-square (RMS) of phase errors in Fig. 4(b)-4(d) are 0.163, 0.083 and 0.038 respectively, which demonstrates the validity of proposed error compensation approach.

\subsection{Experimental results}

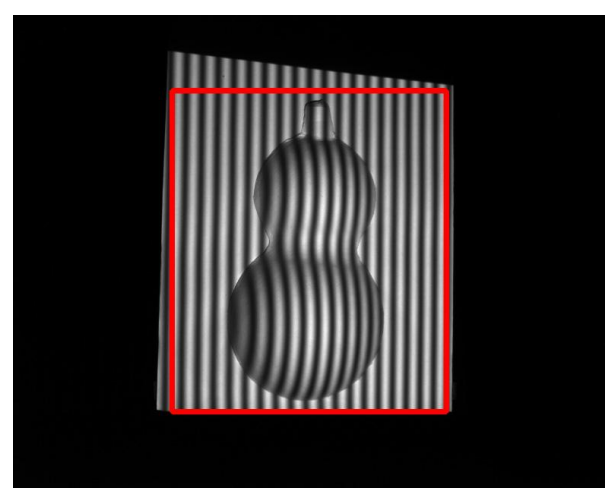

(a)

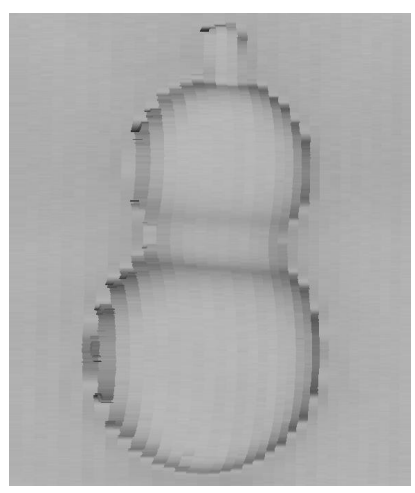

(b)

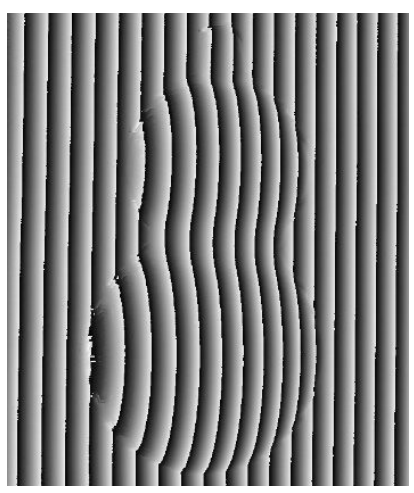

(c) with original contrast

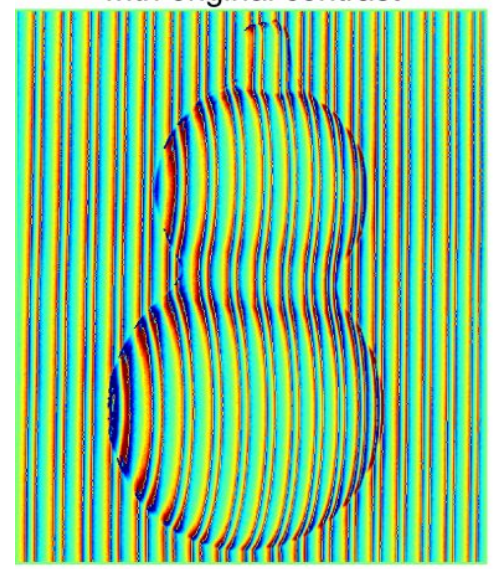

with estimated contrast

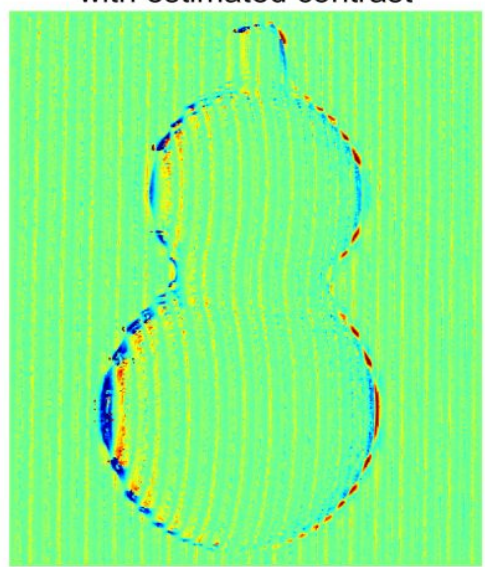

after optimization

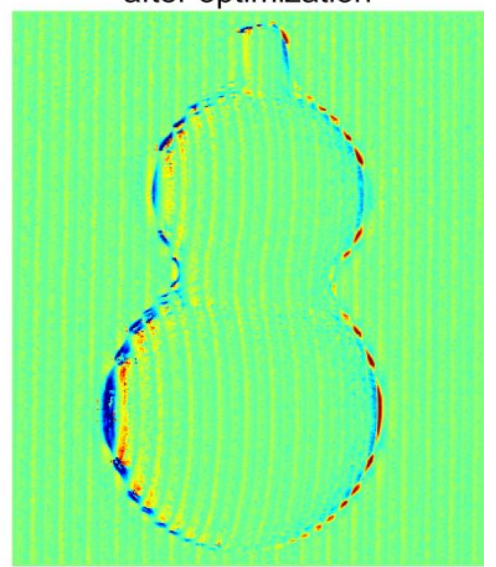

phase error / rad

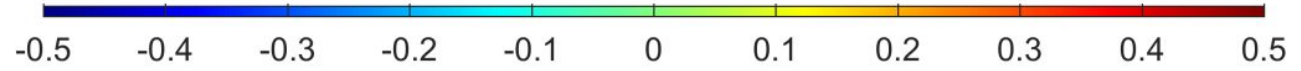

(d)

Figure 6. Experimental results. (a) One of captured fringe images, (b) The estimated fringe contrast, (c) The reconstructed wrapped phase, (d) The comparison of phase errors.

A fringe projection system consisting of a monochrome camera (Basler acA1300-30gm, resolution 1280×1024) and a digital projector (TI DLP LightCrafter 4500, resolution $1140 \times 912$ ) was used. Two sinusoidal fringes with a phase shift of $3 \pi / 2$ are generated by the computer, where $a=0.5, A_{m}=0.8$ and $f=1 / 24$. The patterns are projected onto the surface 
of the object and the camera captures the corresponding images $I_{1}$ and $I_{2}$. In addition, fringe images of 12-step phase-shifting are also captured, with which the phase distribution is calculated with standard phase-shifting algorithm and serves as a criterion to evaluate the phase error of the proposed approach. The experimental results are shown in Fig. 6. Figure 6(a) is one of captured fringe images, and only the region of interest (ROI) marked with red rectangle is processed. The estimated fringe contrast and finally wrapped phase are shown in Fig. 6(b) and 6(c) respectively. Figure 6(d) comparatively shows the distributions of the phase error corresponding to the different stages of the proposed approach. When calculating the phase with the original contrast $A_{m}=0.8$, there are significant phase errors with a periodic distribution, and the RMS of the phase error is 0.227 . If calculating with the estimated fringe contrast, we can get smaller phase error, which has a RMS of 0.070. After the final optimization, the RMS of phase errors has dropped to 0.062. Compared to the simulation results, error reduction after optimization is not so significant in our experimental results. One of the possible reasons is that large errors around the edge of the object that have more influence on the result.

\section{CONCLUSION}

In summary, a two-step phase-shifting algorithm is proposed for phase reconstruction in fringe projection profilometry. The deformed fringe image is formulated based on the working process of fringe projection, and a phase shift of $3 \pi / 2$ is introduced to get the two-step phase-shifting. Considering the intensity ratio between the two fringe images and applying appropriate variable substitution, the process of solving the equations can be regarded as searching the intersection between a line and a circle. With this circle-line model, the number and range of the solutions are analyzed. Afterwards the phase error due to non-zero ambient light is discussed and an approach of error compensation is proposed based on contrast estimation and non-linear least square optimization. Both simulation and experimental results demonstrate the validity of the proposed approach. The experimental results also reveal some shortcomings, including large phase error

at the edge of the object and high time cost in optimization, which need to be investigated in the future. The influences of fringe parameters, e.g., phase jump, fringe period and ambient light, should also be evaluated in the future.

\section{ACKNOWLEDGEMENTS}

The financial supports from the Natural Science Foundation of China (NSFC) under the grant 61775121, 61675117 and 61701321 and that from the Sino-German Center for Research Promotion (SGCRP) under the grant GZ 1391 are gratefully acknowledged. The support of Humboldt Research Fellowship for Postdoctoral Researchers is also acknowledged.

\section{REFERENCES}

[1] Tutsch, R., Petz, M. and Fischer, M., "Optical three-dimensional metrology with structured illumination," Opt. Eng. 50(10), 101507 (2011).

[2] Simon Chane, C., Mansouri, A., Marzani, F. S. and Boochs, F., "Integration of 3D and multispectral data for cultural heritage applications: Survey and perspectives," Image Vision Comput. 31(1), 91-102 (2013).

[3] Gorthi, S. S. and Rastogi, P., "Fringe projection techniques: Whither we are?," Opt. Lasers Eng. 48(2), 133-140 (2010). 
[4] Gai, S., Da, F. and Dai, X., "Novel 3D measurement system based on speckle and fringe pattern projection," Opt. Express 24(16), 17686-17697 (2016).

[5] Wang, M., Yin, Y., Deng, D., Meng, X., Liu, X. and Peng, X., "Improved performance of multi-view fringe projection 3D microscopy," Opt. Express 25(16), 19408-19421 (2017).

[6] Su, X. and Zhang, Q., "Dynamic 3-D shape measurement method: A review," Opt. Lasers Eng. 48(2), 191-204 (2010).

[7] Guan, Y., Yin, Y., Li, A., Liu, X. and Peng, X., "Dynamic 3D imaging based on acousto-optic heterodyne fringe interferometry," Opt. Lett. 39(12), 3678-3681 (2014).

[8] Heist, S., Mann, A., Kühmstedt, P., Schreiber, P. and Notni, G., "Array projection of aperiodic sinusoidal fringes for high-speed three-dimensional shape measurement," Opt. Eng. 53(11), 112208 (2014).

[9] Hu, Y., Chen, Q., Feng, S., Tao, T., Li, H. and Zuo, C., "Real-time microscopic 3D shape measurement based on optimized pulse-width-modulation binary fringe projection," Meas. Sci. Technol. 28(7), 075010 (2017).

[10]Hyun, J.-S., Chiu, G. T. C. and Zhang, S., "High-speed and high-accuracy 3D surface measurement using a mechanical projector," Opt. Express 26(2), 1474-1487 (2018).

[11]Zhang, Z. H., "Review of single-shot 3D shape measurement by phase calculation-based fringe projection techniques," Opt. Lasers Eng. 50(8), 1097-1106 (2012).

[12]Zhong, J. and Weng, J., "Spatial carrier-fringe pattern analysis by means of wavelet transform: Wavelet transform profilometry," Appl. Opt. 43(26), 4993-4998 (2004).

[13]Zhang, Q. and Su, X., "High-speed optical measurement for the drumhead vibration," Opt. Express 13(8), 3110-3116 (2005).

[14] Yin, Y., Wang, M., Gao, B. Z., Liu, X. and Peng, X., "Fringe projection 3D microscopy with the general imaging model," Opt. Express 23(5), 6846-6857 (2015).

[15]Flores, J. L., Ferrari, J. A., García Torales, G., Legarda-Saenz, R. and Silva, A., "Color-fringe pattern profilometry using a generalized phase-shifting algorithm," Appl. Opt. 54(30), 8827-8834 (2015).

[16]Zheng, D., Da, F., Kemao, Q. and Seah, H. S., "Phase-shifting profilometry combined with gray-code patterns projection: Unwrapping error removal by an adaptive median filter," Opt. Express 25(5), 4700-4713 (2017).

[17]Zhang, S., "Recent progresses on real-time 3D shape measurement using digital fringe projection techniques," Opt. Lasers Eng. 48(2), 149-158 (2010).

[18] Quan, C. G., Tay, C. J., Kang, X., He, X. Y. and Shang, H. M., "Shape measurement by use of liquid-crystal display fringe projection with two-step phase shifting," Appl. Opt. 42(13), 2329-2335 (2003).

[19]Hui, T. W. and Pang, G. K. H., "3-D measurement of solder paste using two-step phase shift profilometry," IEEE Trans. Electron. Packag. Manuf. 31(4), 306-315 (2008).

[20] Yang, F. and He, X., "Two-step phase-shifting fringe projection profilometry: Intensity derivative approach," Appl. Opt. 46(29), 7172-7178 (2007).

[21] Meng, X. F., Peng, X., Cai, L. Z., Li, A. M., Guo, J. P. and Wang, Y. R., "Wavefront reconstruction and three-dimensional shape measurement by two-step dc-term-suppressed phase-shifted intensities," Opt. Lett. 34(8), 1210-1212 (2009).

[22] Weise, T., Leibe, B. and Van Gool, L., "Fast 3D scanning with automatic motion compensation," Proc. IEEE Conference on Computer Vision and Pattern Recognition 2007 (CVPR '07), 1-8 (2007). 